
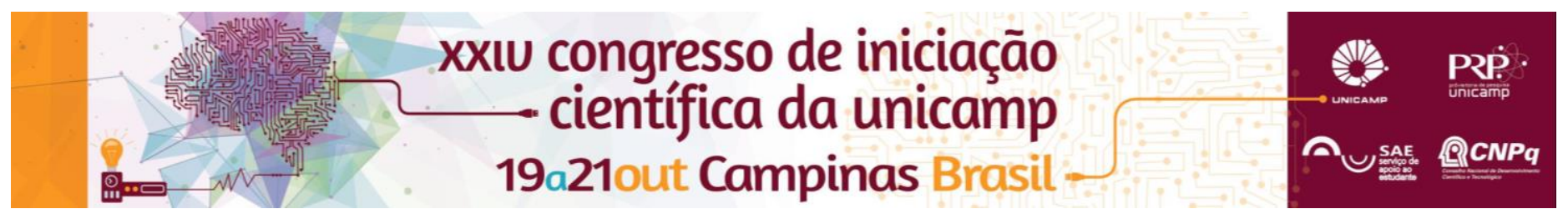

\title{
Comparative study between Experiments and numerical simulation (CFD) of interfacial waves characteristics in wavy stratified oil-water flow pattern in pipes.
}

\author{
André G. D. de Arroxellas*, Marcelo S. de Castro, Ricardo P. Ávila.
}

\begin{abstract}
Two-phase flows consist in the, physical and chemical, immiscibility of transported fluid components that arrange in phases. These phases, or fractions of fluids, may be disposed into two-phase flows such as: oil and water; oil and air, by the fluid at different phases, or liquid and vapor. The particular patterns in which these flows are geometrically arranged depend on flow rates of phases, pressures, temperatures, fluid properties, among others. These intrinsic characteristics construct a, yet growing, data base for Engineers to stablish connections between experimental data and computational simulation (CFD-Computational Fluid Dynamics) and avoid industrial problems, like in cases of oil elevation, subsea flow and transportation chain. The proposed work is the sole sprout of this conjunction, that intends to analyze interfacial waves characteristics through videos of stratified oil-water flows.
\end{abstract}

\section{Key words: \\ Two-phase flows, Stratified oil-water flow, Characteristics of interfacial waves.}

\section{Introduction}

The recent subsea technology in Petroleum Industry associated with the advent of more powerful processors, lenses and sensors corroborate to bring forth research efforts to investigate and consolidate, through mathematical models and laboratory data, the art of physical phenomena involved in fluid transportation.

The prerogative of this work is to propose an applicability of image treatment algorithms in order to extract laboratory data and prove applied techniques in CFD (Computational Fluid Dynamics).

\section{Results and Discussion}

Every algorithm consists in a series of specific routines designed to solve a notorious case. This fact demands an adaptability of code in order to analyze a sequence of images. Real images require greater attention due to pre-treatment need. Filters and transforms, such as Gauss, Hugh and distance are, then, applicable. Nonetheless the morphologic essence is almost invariable for every flow seen at its side.

\section{Essential Routine:}

- Through a small element is defined and catalogued the regions, molded by the element, that are connected to each other.

- A structural element permits a close morphology (dilation followed by erosion).

$$
A \cdot B=(A \oplus B) \ominus B
$$

- An histogram-based threshold is applied.

Some features may be necessary so that every step of the routine coexist, such as: elimination of disconnected regions, set-up of region of interest, experiment light normalization etc.

These settings proved to be sufficient in the cases of oil-water stratified flows and numerical simulations, exposing fractions, height and velocity of the phases as represented in the images bellow:
Image 1. Didactically selected side and frontal wave interfaces, excluding disconnected regions, post morphological processing (Arroxellas, Ávila and Castro, 2016).

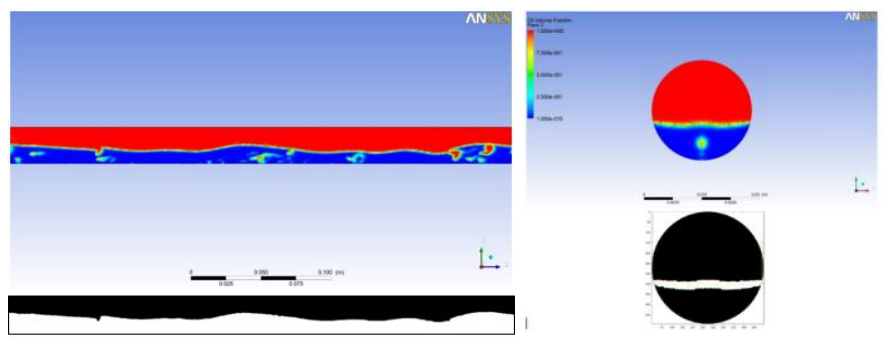

\section{Conclusions}

The applicability of the presented methods is consistent and adaptable to many real situations directly. The understanding of image treatment permits to verify and evaluate experimental and laboratorial data, granting to image acquisition, when environmentally possible, methodology excellence. Notwithstanding the environment and equipment set-up are, although simplistic, crucial to develop a reliable pre-treatment phase.

\section{Acknowledgement}

This work was only achieved with the collaboration of: Phd. Marcelo Souza de Castro; Phd. Ricardo P. Ávila and MSc. Cléber C. Pereira, to whom I'm grateful.

I also thank the PIBIC and CNPq initiatives to support the research at UNICAMP.

\footnotetext{
1 Dougherty, E. R. and Lotufo R. A., Hands-on Morphological Image Processing. In Tutorial Texts in Optical Engineering, Volume TT59; 2003, SPIE: Florida, pp 27-29 and pp 66-68.

${ }^{2}$ Castro, M. S. de Pereira; C. C. Pereira and Santos J. N. dos, Geometrical and kinematic properties of interfacial waves in horizontal heavy oil-water stratified flow. In computational Methods in Multiphase Flow VI; 2011, : WIT press, pp 227-238.
} 